\title{
Profile of Ipragliflozin, an Oral SGLT-2 Inhibitor for the Treatment of Type 2 Diabetes: The Evidence to Date
}

\section{Wajd Alkabbani $\mathbb{C}$ John-Michael Gamble (iD}

School of Pharmacy, Faculty of Science, University of Waterloo, Kitchener, ON, Canada
Correspondence: John-Michael Gamble School of Pharmacy, Faculty of Science, University of Waterloo, I0A Victoria

Street S, Kitchener, ON, N2G IC5 Canada

Tel + I 5198884567

$\mathrm{Fax}+I 5198837580$

Email jm.gamble@uwaterloo.ca
Background: Sodium-glucose cotransporter-2 (SGLT-2) inhibitors are a novel class of pharmacotherapeutics for type 2 diabetes management that work by reducing renal reabsorption of glucose. Ipragliflozin is a potent, selective SGLT-2 inhibitor used for the management of type 2 diabetes.

Objective: The primary aim of this review is to summarize the available evidence on the efficacy and safety of ipragliflozin for the management of type 2 diabetes. We also review the discovery, pharmacokinetic, and pharmacodynamic profile of ipragliflozin.

Methods: To inform our review, we searched MEDLINE, International Pharmaceutical Abstracts, and Embase to identify relevant papers to ipragliflozin use in type 2 diabetes. Clinical trial registries were also searched.

Results: Findings from randomized clinical trials demonstrate that compared to placebo, ipragliflozin significantly reduces glucose as measured via Hemoglobin A1c and fasting plasma glucose levels. Ipragliflozin is also associated with weight reduction and an improvement in some, but not all, cardiovascular risk markers. Ipragliflozin has a favourable safety profile with a low risk of hypoglycemia and the rates of common adverse events are not significantly different than placebo. Limited data are available to assess rare and long-term adverse effects.

Conclusion: Current evidence shows that ipragliflozin is an effective therapeutic option for the management of glucose control in type 2 diabetes. However, no cardiovascular outcome trials have been conducted to date. Real-world observational studies are still needed to accurately capture any possible rare or long-term adverse events.

Keywords: review, efficacy, safety, pharmacokinetics, pharmacodynamics, discovery

\section{Introduction}

Type 2 diabetes mellitus (T2D) is a metabolic disorder that results from pancreatic beta cell ( $\beta$-cells) dysfunction to secret insulin and/or the failure of insulin to exert its biological influence at the level of peripheral tissues (eg, muscle, adipose, or hepatic tissues). ${ }^{1}$ The latter is a resistance to insulin action that is accompanied by an inadequate compensatory response of insulin secretion. Overtime, this results in longstanding hyperglycemia and disturbances in the metabolism of carbohydrate, fat, and protein with consequences resulting in long-term complications affecting both micro- and macro-vasculature. ${ }^{1}$ The risk of developing T2D increases with several factors, such as age, obesity, family history, lack of physical activity, presence of related comorbidities like hypertension, as well as certain drugs (eg, glucocorticoids). ${ }^{2}$ 
The characteristic hallmark of T2D is hyperglycemia and is a consequence of multiple pathogenic abnormalities. The term "'ominous octet" has been coined to describe the various pathogenic mechanisms involved, including decreased insulin secretion, decreased incretin effect, decreased glucose uptake, neurotransmitter dysfunction, increased lipolysis, increased glucagon secretion, increased hepatic glucose production, and increased glucose reabsorption. ${ }^{3}$ Targeting these pathophysiologic defects is a key principle in the pharmacologic management of T2D. Indeed, the development of a novel class of antihyperglycemic agents, called the sodium-glucose cotransporter-2 (SGLT-2) inhibitors, allows for targeting of the increased glucose reabsorption in the kidney that occurs in T2D. ${ }^{4}$ This novel mechanism of action is independent of insulin secretion and sensitivity, making this class complementary to most current diabetes therapies.

The US Food and Drug Administration (FDA) approved canagliflozin in 2013, followed by nine additional SGLT-2 inhibitors becoming approved internationally. ${ }^{5}$ One of the SGLT-2 inhibitor molecules is ipragliflozin (ASP-1941), which is jointly produced by the Japanese companies, Astellas Pharma Inc. and Kotobuki Pharmaceutical, under the brand name Suglat ${ }^{\circledR}$. ${ }^{6}$ Ipragliflozin was the first SGLT-2 inhibitor to obtain regulatory approval in Japan on January 17, 2014 and was subsequently approved in Russia and South Korea. ${ }^{6,7}$ The aim of this article is to review the profile of ipragliflozin in respect to its discovery, pharmacokinetics, pharmacodynamics, clinical efficacy, safety, and role in the management of type 2 diabetes.

\section{Methods}

\section{Literature Search and Screening}

To inform our review we conducted a comprehensive search of the evidence base. First, a literature search was conducted on January 22, 2021, using the following databases: Embase (1974 to 2021 January 20), International Pharmaceutical Abstracts (1970 to January 2020), and Ovid MEDLINE (including Epub Ahead of Print, In-Process \& Other NonIndexed Citations, Daily and Versions(R) from 1946 to January 20, 2021). The OVID search strategy included ipragliflozin as a key term and Emtree term, Suglat as a key term, as well as other relevant identifiers for ipragliflozin such as ASP1941, accession number (DB11698), CAS registry number (761423-87-4), and unique ingredient number [UNII] (3N2N800R7X). The search results were limited to English language citations and humans.
Clinicaltrials.gov and Japan's University hospital Medical Information Network-Clinical Trial Registry (UMIN-CTR) were also searched for ongoing and completed trials for ipragliflozin. Second, citations were imported into Covidence review software. ${ }^{8}$ Third, we screened titles and abstracts for relevant citations followed by full-text screening. Fourth, we classified included citations according to main topic (Chemistry/biochemistry, pharmacokinetics and/ or pharmacodynamics, efficacy and safety measures, multiple topics, or other) and study design (eg, RCT, observational, preclinical, review) to aid in the writing of this review. Citations focused on ipragliflozin for the management of type 1 diabetes were excluded as they were considered out of scope for this review.

\section{Efficacy and Safety Assessment}

To summarize the best available evidence on the clinical efficacy and safety of ipragliflozin, we further limited our review to include Phase 3 and 4 double blinded RCTs with placebo or active comparator. We also integrate evidence from currently available systematic reviews and metaanalyses. The primary efficacy outcomes of interest were change in 1) HbA1c, 2) fasting blood glucose (FPG), 3) systolic and diastolic blood pressure (SBP and DBP), and 4) body weight. We also assessed changes in relevant metabolic parameters, including uric acid, and lipids (LDL, HDL, triglycerides). The primary safety outcomes were 1) hypoglycemia, 2) genital and urinary tract infections (GTI and UTI), 3) acute kidney injury (AKI), 4) diabetic ketoacidosis (DKA), 5) bone fractures, 6) amputations, and 7) skinrelated adverse events. These adverse events have either been linked with SGLT-2 inhibitors as a class or are listed as "adverse reactions likely to be encountered" by the Japan Diabetes Association's recommendation on the proper use of SGLT-2 inhibitors. ${ }^{9}$ Using a standardized form, we also extracted the mean difference (SD) for the continuous efficacy outcomes and the relative risks $(95 \%$ Confidence interval) for the dichotomous safety outcomes.

This review relied exclusively on anonymous information published in the peer-reviewed literature or publicly available and therefore did not require ethics approval from the University of Waterloo health ethics review board.

\section{Results}

\section{Studies on Ipragliflozin}

There were over 900 non-duplicate citations identified from our search strategy, of which 166 were deemed 
relevant for this review. Of these, 5 were pertaining to chemistry as a main topic, 41 to pharmacokinetics and/or pharmacodynamics, 111 to efficacy and safety, and 9 to multiple or other topics. Most studies designs were RCTs $(\mathrm{n}=64)$, followed by single-arm interventional design $(\mathrm{n}=22)$. Only 15 were mechanistic/preclinical studies, 11 were review articles, 6 were systematic reviews, 4 were meta-analyses, 12 were case reports, 3 were letters or editorials, 4 were cohort studies, 1 was a case-control and 1 was cross-sectional study. A total of 23 designs were considered as other or unclear design.

\section{Discovery and Chemical Properties}

Phlorizin (Figure 1A) is a natural O-glucoside potent nonselective SGLT inhibitor. However, due to its poor metabolic stability and SGLT-1 inhibitory activity, it was not considered a suitable drug candidate. ${ }^{10}$ This led to the development of C-glucoside derivatives (Figure 1B), which were found to be metabolically more stable than O-glucosides. ${ }^{10}$ Imamura et al investigated the effects of replacing the terminal benzene ring in the $\mathrm{C}$-glucoside derivatives with various heteroaromatics, including a benzothiophene (Figure 1C). ${ }^{10}$ This resulted in a potent SGLT-2 inhibitor (Inhibitory Concentration $[\mathrm{IC}]_{50}=30 \mathrm{nM}$ ) with a good selectivity versus SGLT- $1 .{ }^{10}$ Further optimization, with a fluorine atom substitution (Figure 1D) resulted in ipragliflozin, which possess a better in vitro profile $\left(\mathrm{IC}_{50}=7.4 \mathrm{nM}\right)$ and a 254 -fold selectivity for SGLT-2 versus SGLT- $1 .{ }^{10}$ Ipragliflozin, or (1S)-1,5-anhydro-1-C-\{3-[(1-benzothiophen-2-yl) methyl]4-fluorophenyl\}-D-glucitol, is a relatively small molecule (Molecular Weight $=404.5 \mathrm{~g} / \mathrm{mol}$ ), moderately lipophilic, and soluble in organic solutions, such as ethanol $(30 \mathrm{mg} / \mathrm{mL})$ but sparingly soluble in aqueous solutions $(0.0299 \mathrm{mg} / \mathrm{mL}){ }^{11-13}$

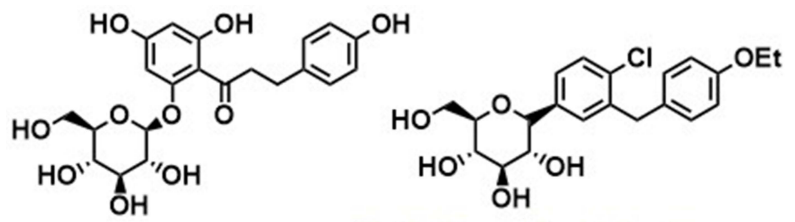

1A. Phlorizin
1B. C-Glucoside derivative eg: Dapagliflozin

\section{Pharmacokinetics}

Ipragliflozin is suggested to exhibit dose-dependent pharmacokinetics. It is rapidly absorbed after oral administration, reaching peak concentrations $\left(\mathrm{C}_{\max }\right)$ within $\left(\mathrm{t}_{\max }\right)$ 0.5-2.5 hours. ${ }^{14-16}$ Oral bioavailability is reported as $90 \%$ by Astellas. ${ }^{17}$ In plasma, ipragliflozin is $94.6-96.5 \%$ bound to plasma proteins, with albumin as the main binding partner. ${ }^{16}$ Nevertheless, it achieves extensive extravascular distribution. The plasma concentration-time profile is typically biphasic, and the terminal plasma half-life is estimated to be $10-15$ hours. ${ }^{16}$ With increasing doses, the maximum concentration after oral administration and overall exposure (AUC) have been reported to increase in a slightly-less-than-proportional manner. ${ }^{16}$ Similar to other SGLT-2 inhibitors, the metabolism of ipragliflozin to inactive metabolites is primarily accomplished via glucuronidation, with major contribution from the UGT2B7 isoform and minor contribution from the UGT2B4 and UGT1A9 isoforms. ${ }^{16}$ Notable amounts of unchanged ipragliflozin can be recovered from feces, suggesting that biliary excretion of unchanged drug may play a role in its elimination. ${ }^{16}$ Only $1-2 \%$ of the orally administered dose is excreted unchanged in urine (renal clearance $=0.09$ to $0.13 \mathrm{~L} /$ h), but tubular concentrations of free drug have been reported as adequate for therapeutic effect. ${ }^{16}$

The effects of age and gender on the pharmacokinetics and pharmacodynamics of ipragliflozin were examined in a Phase I, double-blind, placebo-controlled RCT. Peak concentrations $\left(\mathrm{C}_{\max }\right)$ and steady-state exposures $\left(\mathrm{AUC}_{24}\right)$ are slightly higher in females and in older subjects ( $>65$ years). After multiple oral doses of ipragliflozin, among females, concentration was higher for participants 65 years and older compared to younger participants, geometric mean ratio [GMR] (90\% confidence interval $[\mathrm{CI}])$ was $1.45(1.27,1.67)$ for $\mathrm{AUC}_{24}$ and was $1.25(1.06,1.49)$ for $\mathrm{C}_{\max }$. Among males, GMR for $\mathrm{AUC}_{24}$

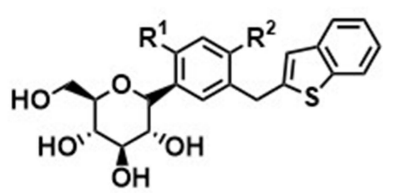

1C. Benzothiophene derivatives

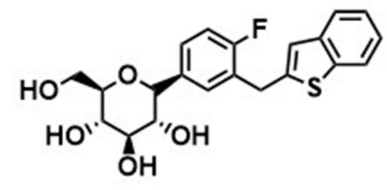

1D. Ipragliflozin

Figure I Chemical structure of phlorizin and derivatives: (A) Phlorizin, (B) C-glucoside derivative, (C) Benzothiophene derivative, (D) Ipragliflozin. 
was $1.21(1.06,1.38)$ for older compared to younger participants; however, the $\mathrm{C}_{\max }$ was similar. Compared to males, females had higher concentrations of ipragliflozin $\operatorname{GMR}(90 \% \mathrm{CI})=1.39(1.22,1.59)$ for $\mathrm{AUC}_{24}$ and 1.35 $(1.15,1.58)$ for $\mathrm{C}_{\max }$, among those 65 years and older. ${ }^{16}$

Despite only $\sim 1 \%$ of total clearance being attributed to renal excretion, peak concentrations of ipragliflozin and overall exposures $\left(\mathrm{AUC}_{\infty}\right)$ are increased in those with moderate and severe renal impairment. In a Japanese study $(\mathrm{n}=25), \mathrm{C}_{\max }$ and $\mathrm{AUC}_{\infty}$ were 1.17 times and 1.21 times higher, respectively, in T2D patients with moderate renal impairment compared to patients with normal kidney function. $^{18}$ Similarly, in a Europe-based study $(n=32)$ $\mathrm{AUC}_{\infty}$ increased by 1.40 and 1.47 in T2D patients with moderate and severe renal impairment, respectively. ${ }^{19}$ These observations may be on account of reduced ipragliflozin metabolism by UGT2B7 in the kidney, as both kidney volume and renal perfusion are known to decrease in patients with poor renal function. ${ }^{16}$ The major metabolite (M2) significantly accumulated in patients with moderate and severe renal impairment. ${ }^{16}$ The $\mathrm{AUC}_{\infty}$ of $\mathrm{M} 2$ increased by 2.1-3.0 times in patients with moderate impairment and by 3.3-5.4 times in patients with severe impairment compared to those with normal renal function. ${ }^{16}$ These changes were considered modest and adjustment of the daily dose of ipragliflozin is not necessary in the presence of chronic kidney disease. ${ }^{20}$

On the contrary, moderate hepatic impairment was found to have no clinically relevant effects on the pharmacokinetics of ipragliflozin or its major metabolite (M2). There were no clear differences observed in the half-life or protein binding of ipragliflozin between those with moderate hepatic impairment and normal subjects. For M2, $\mathrm{C}_{\max }$ and $\mathrm{AUC}_{\infty}$ were similar for both groups. ${ }^{16}$

\section{Pharmacodynamics}

The kidneys play a role in maintaining glucose homeostasis by filtering and reabsorbing glucose. Glucose is first filtered in the glomeruli in the nephron and is then reabsorbed in the proximal tubules. ${ }^{21,22}$ In a healthy adult, the kidneys filter approximately 160 to 180 grams of glucose per day on average, of which about $99 \%$ is reabsorbed by the kidney. ${ }^{21,23}$ However, due to the higher renal glucose reabsorption capacity in $\mathrm{T} 2 \mathrm{D}$, excess glucose that is excreted in the urine under normal physiological conditions is reabsorbed, exacerbating hyperglycaemia. ${ }^{24}$ The SGLT are membrane proteins expressed in the intestine and proximal tubules of the kidney and are responsible for the transport of glucose, as well as amino acids, vitamins, and electrolytes. $^{23,25}$ Specifically, the low affinity, high capacity SGLT-2 proteins, located on the S1 and S2 segments of the proximal convoluted tubule reabsorb about $90 \%$ of the filtered glucose, whereas the high affinity, low capacity SGLT-1 proteins reabsorb the remaining $10 \% .^{26,27}$ In humans without T2D the maximal transport capacity of glucose per day is about 450 to 500 grams and the plasma glucose threshold is approximately 10 to 11 $\mathrm{mmol} / \mathrm{L}{ }^{28}$ However, in persons with $\mathrm{T} 2 \mathrm{D}$, the maximal transport capacity is increased up to 600 grams of glucose per day. ${ }^{28}$ Based on animal models, it is thought that SGLT-2 proteins are overexpressed in patients with T2D and thereby increase the maximal glucose transport capacity and decrease the plasma glucose threshold. ${ }^{28}$ Ipragliflozin exerts its effect by selectively inhibiting the SGLT-2 protein, thereby increasing urinary glucose excretion. ${ }^{16}$ Indeed, the inhibition of the SGLT-2 protein induces about 50 to 80 grams (varies by dose and SGLT-2 inhibitor molecule) of urinary glucose excretion per day. ${ }^{29}$ Therefore, the main pharmacodynamic effect of ipragliflozin is through increased glucose excretion leading to the main desired clinical effect of decreased plasma glucose concentration.

In healthy Japanese participants, a dose-dependent increase in urinary glucose excretion over 24-hour dosing interval $\left(\mathrm{UGE}_{24}\right)$ has been reported after a single-dose administration. The maximum $\mathrm{UGE}_{24} \quad(\approx 50 \mathrm{~g})$ was approached after the final dose in a 7-day $50 \mathrm{mg}$ multiple dosing regimen. ${ }^{16}$ Similarly, in healthy European participants, ipragliflozin also increased $\mathrm{UGE}_{24}$ in a dose-dependent manner (Max $\mathrm{UGE}_{24} \approx 59 \mathrm{~g}$ ) following multiple doses. ${ }^{19}$ In both studies, plasma glucose levels $\left(\mathrm{AUC}_{24}\right)$ in healthy subjects were not affected.

In T2D Japanese patients, the change in $\mathrm{UGE}_{24}$ after 14 days was higher for ipragliflozin $50 \mathrm{mg}$ /day (mean change (SD) in $\mathrm{UGE}=80.6$ (22.2)) compared to placebo (mean change in UGE $=5.3(19.3)$ ), in a double-blinded RCT. ${ }^{14}$ Additionally, the change in plasma glucose $\mathrm{AUC}_{24}$ was -1103.7 (639.9) for ipragliflozin compared to placebo 63.8 (732.5). $^{14}$

Interestingly, despite higher concentrations and greater drug exposure among females and those 65 years and older, the change in $\mathrm{UGE}_{24}$ was lower in these same subpopulations, GMR $(90 \% \mathrm{CI})=0.69(0.59,0.80)$ for older vs younger and $0.71(0.61,0.82)$ for females vs males. ${ }^{16}$ This observation may be attributed to lower glomerular filtration of unchanged drug into the tubular 
filtrate, as UGE was previously shown to be correlated in a direct linear proportion with glomerular filtration rate (eGFR) in patients with otherwise similar ipragliflozin exposures and baseline fasting plasma glucose levels. The magnitude of the differences did not warrant a dose adjustment. $^{16}$

Similarly, this phenomenon is also demonstrated in renal impairment, as the change in $\mathrm{UGE}_{24}$ in $\mathrm{T} 2 \mathrm{D}$ patients with moderate renal impairment was only $54 \%$ of that in patients with normal renal function, despite higher exposure in T2D patient with renal impairment. ${ }^{16}$ Nevertheless, the efficiency of ipragliflozin action (fractional glucose excretion) was maintained in patients with renal impairment. $^{20}$ Lastly, no clear differences were observed in the $\mathrm{UGE}_{24}$ between those with moderate hepatic impairment compared to normal hepatic function in an openlabel, single-dose, parallel-group study conducted in the USA. $^{30}$

\section{Clinical Efficacy of Ipragliflozin}

Herein, we summarize the evidence of ipragliflozin efficacy for multiple end points relevant to T2D. Our review is primarily based on evidence from 10 Phase III and IV double-blinded randomized controlled trials which are summarized in Table 1. ${ }^{30-40}$ In addition, we considered results from published quantitative systematic reviews reporting on various efficacy and safety end points. ${ }^{7,41-44}$

\section{Glycemic Control}

All included trials assessed change from baseline in HbA1c level as a primary outcome and FPG level as a secondary outcome, albeit at different time points. The mean difference of change in HbA1c level from baseline was significant in all studies and ranged from $-0.24 \%$ to $1.30 \%$ favouring ipragliflozin over placebo (Figure 2A). Similarly, the mean difference of the change in FPG levels ranged from -8.20 to $-46.50 \mathrm{mg} / \mathrm{dL}$, favoring ipragliflozin (Figure 2B). Notably, results from LANTERN trial showed that ipragliflozin significantly improved glycemic control in T2D patients with mild renal impairment but did not improve glycemic control in those with moderate renal impairment. $^{34}$

These results are consistent with previous quantitative systematic reviews which used different eligibility criteria for study inclusion in their meta-analysis. ${ }^{41,43}$ For example, Elgebaly et al meta-analysed 13 RCTs $(n=2535$ patients) and found that ipragliflozin $50 \mathrm{mg}$ as monotherapy, compared to placebo, reduced HbAlc by $1.20 \%(95 \%$
CI $-1.47,-0.93)$ and FPG by $1.30 \mathrm{mg} / \mathrm{dL}(95 \% \mathrm{CI}-1.93$, $-0.67)$. Ipragliflozin $50 \mathrm{mg}$ as add-on therapy to metformin, sulfonylurea, pioglitazone, and insulin also reduced plasma glucose as measured by $\mathrm{HbAlc}$ and FPG. ${ }^{41}$

\section{Blood Pressure Reduction}

Systolic and diastolic blood pressure were assessed as secondary outcomes in eight out of the 10 included trials; however, one trial did not report data for diastolic blood pressure. ${ }^{36}$ There were no differences in the change of systolic and diastolic blood pressure between ipragliflozin and placebo in most included trials (Figure 3A and B). Although a 2017 meta-analysis of six trials reported a significant reduction in both systolic and diastolic blood pressures for ipragliflozin $50 \mathrm{mg}$ vs placebo, ${ }^{42}$ a larger 2018 meta-analysis did not find any differences in blood pressure and was consistent with our findings. ${ }^{41}$

\section{Body Weight Reduction}

All trials significantly favoured ipragliflozin group over placebo group in body weight reduction, with mean differences in weight change ranging from -1.04 to $-2.80 \mathrm{~kg}$ (Figure 4). This reduction in body weight is consistent with a previous meta-analysis. ${ }^{41}$

\section{Other Metabolic Effects}

A previous meta-analysis reported improvement in several additional cardiometabolic parameters including highdensity lipoprotein, triglycerides, and uric acid. ${ }^{43}$ Similarly, among our included studies reporting change in lipid parameters $(n=7)$, most studies reported a mean increase in high-density lipoprotein of 0.8 to $5.20 \mathrm{mg} / \mathrm{dL}$, favouring ipragliflozin (Figure S1.A); however, there was no significant change in low-density lipoprotein (Figure


reduction in triglycerides by most studies (Figure S1.C). Only 1 of our included studies reported uric acid which was similar in the ipragliflozin and placebo groups. ${ }^{32}$

\section{Cardio-Renal Effects}

Although there have been landmark clinical trials reporting cardiovascular and renal benefits of several SGLT-2 inhibitors including empagliflozin (EMPA-REG OUTCOME), ${ }^{45}$ canagliflozin (CANVAS PROGRAM), ${ }^{46}$ dapagliflozin (DELCARE-TIME 58), ${ }^{47}$ and ertugliflozin (VERTIS), ${ }^{48}$ and sotagliflozin (SCORED), ${ }^{49}$ a large cardiovascular outcome trial has not been conducted for ipragliflozin. It is generally thought that the cardio-renal benefits of SGLT-2 inhibitors are a class effect. Briefly, SGLT-2 


\begin{tabular}{|c|c|c|c|c|c|c|c|c|c|c|}
\hline 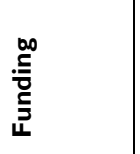 & 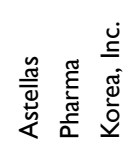 & 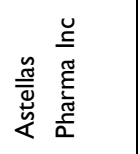 & 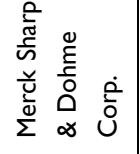 & 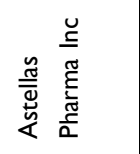 & 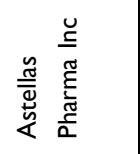 & 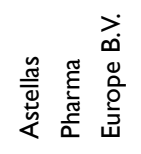 & 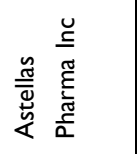 & 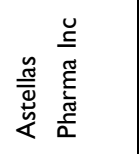 & 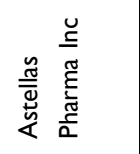 & 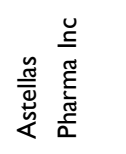 \\
\hline 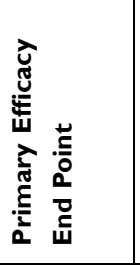 &  & 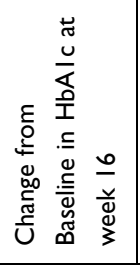 & 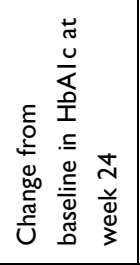 & 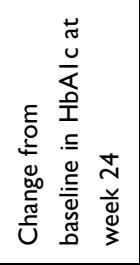 & 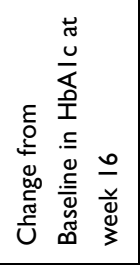 & 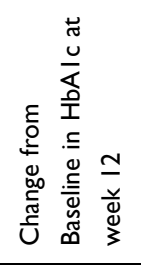 &  &  & 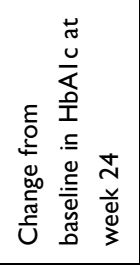 & 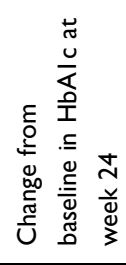 \\
\hline 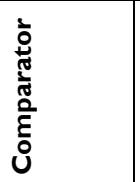 & $\begin{array}{l}\stackrel{\circ}{\mathbb{\Xi}} \\
\frac{\tilde{\pi}}{0}\end{array}$ & $\begin{array}{l}\stackrel{\circ}{\stackrel{\Xi}{u}} \\
\frac{\tilde{\pi}}{\alpha}\end{array}$ & $\begin{array}{l}\stackrel{8}{8} \\
\frac{\mathscr{U}}{0} \\
\frac{\pi}{0}\end{array}$ & $\begin{array}{l}\stackrel{\circ}{\mathbb{\Xi}} \\
\frac{\tilde{\pi}}{0}\end{array}$ & $\begin{array}{l}\stackrel{\circ}{\mathbb{\Xi}} \\
\frac{\tilde{\pi}}{0}\end{array}$ & $\begin{array}{l}\stackrel{\circ}{\mathbb{\Xi}} \\
\frac{\pi}{0}\end{array}$ & 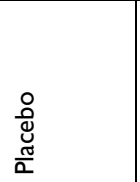 & 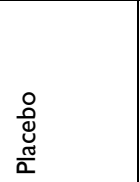 & $\begin{array}{l}\stackrel{\circ}{\mathbb{U}} \\
\frac{\tilde{\pi}}{0}\end{array}$ & $\begin{array}{l}\stackrel{\circ}{\stackrel{\Xi}{0}} \\
\frac{\tilde{\pi}}{0}\end{array}$ \\
\hline 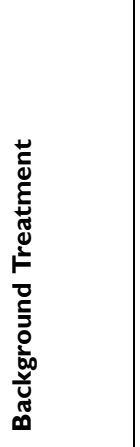 & $\begin{array}{l}\stackrel{0}{0} \\
\stackrel{0}{z}\end{array}$ & $\begin{array}{l}0 \\
\stackrel{0}{0} \\
z\end{array}$ & 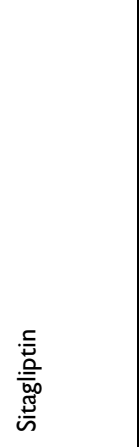 & 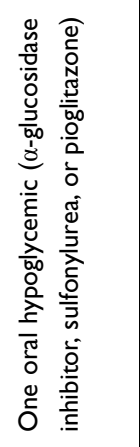 & $\begin{array}{l}\underline{\underline{\overline{\bar{z}}}} \\
\underline{\underline{\underline{\underline{\underline{m}}}}}\end{array}$ & 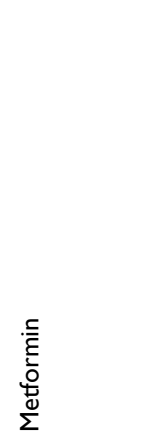 &  & 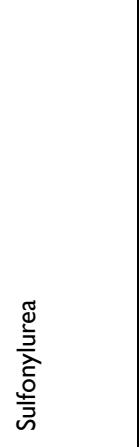 & 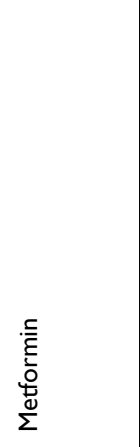 & 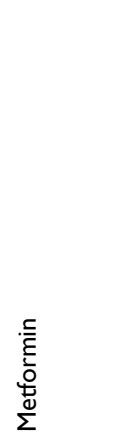 \\
\hline 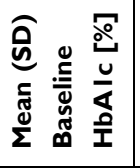 & 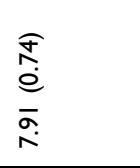 & $\begin{array}{l}\text { E } \\
0 \\
\sim \\
0 \\
\infty \\
0\end{array}$ & $\begin{array}{l}\widehat{\hat{N}} \\
\stackrel{0}{0} \\
\delta \\
\infty \\
\infty\end{array}$ & 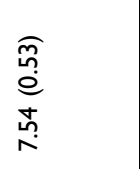 & $\begin{array}{l}\widehat{ఠ} \\
\infty \\
\stackrel{0}{0} \\
0 \\
\infty \\
\infty\end{array}$ &  & $\begin{array}{l}\widehat{o} \\
\stackrel{0}{0} \\
\text { స్ } \\
\infty\end{array}$ & $\begin{array}{l}\hat{\sigma} \\
\stackrel{0}{0} \\
\hat{n} \\
\infty \\
0\end{array}$ & 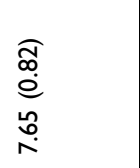 & $\begin{array}{l}\widehat{\widehat{N}} \\
\stackrel{0}{0} \\
\text { స్ } \\
\infty\end{array}$ \\
\hline 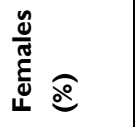 & ڤ̊̀ & $\stackrel{m}{\text { p. }}$ & นี & $\frac{a}{\dot{N}}$ & 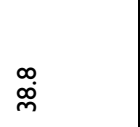 & $\stackrel{\text { : }}{\text { in }}$ & 芫 & $\overline{\dot{m}}$ & f่̊̀ & $\overline{\dot{\sigma}}$ \\
\hline 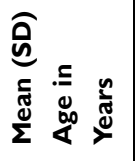 & 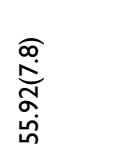 &  &  & 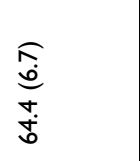 & 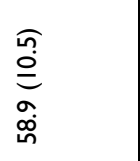 & 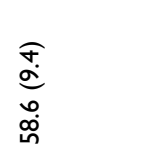 & 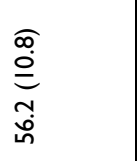 & 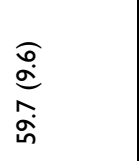 &  & $\begin{array}{l}\text { त̊ } \\
\stackrel{0}{=} \\
\hat{\circ}\end{array}$ \\
\hline 豆 & $\stackrel{\stackrel{\rho}{\underline{m}}}{ }$ & $\stackrel{\Xi}{\underline{\simeq}}$ & $\stackrel{\tilde{q}}{\underline{q}}$ & $\underline{ \pm}$ & 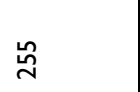 & $\underline{\underline{0}}$ & $\underline{\underline{n}}$ & $\stackrel{?}{d}$ & $\underline{\Omega}$ & 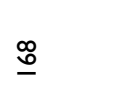 \\
\hline 䨔 & 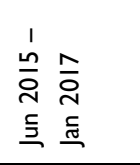 &  & 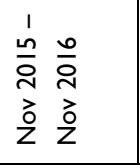 & 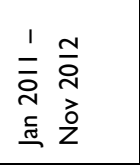 & 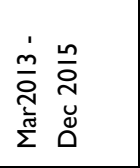 & 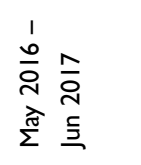 & 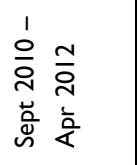 & 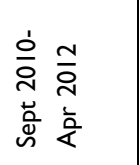 &  & 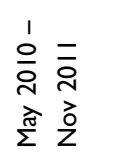 \\
\hline لَّ &  & 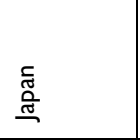 & 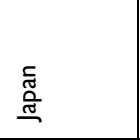 & 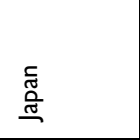 & $\begin{array}{l}\text { 总 } \\
\text { 恖 }\end{array}$ &  &  & 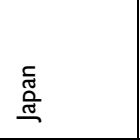 & 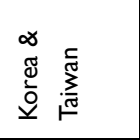 & 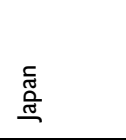 \\
\hline 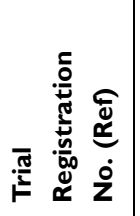 & 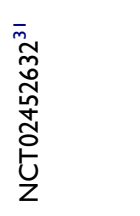 &  & 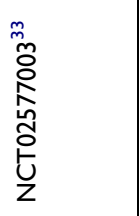 & 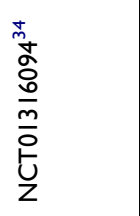 & 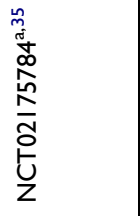 & 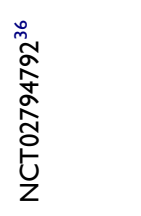 & 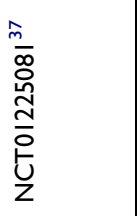 & 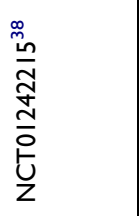 &  & 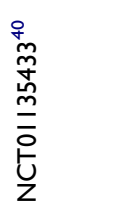 \\
\hline
\end{tabular}


A

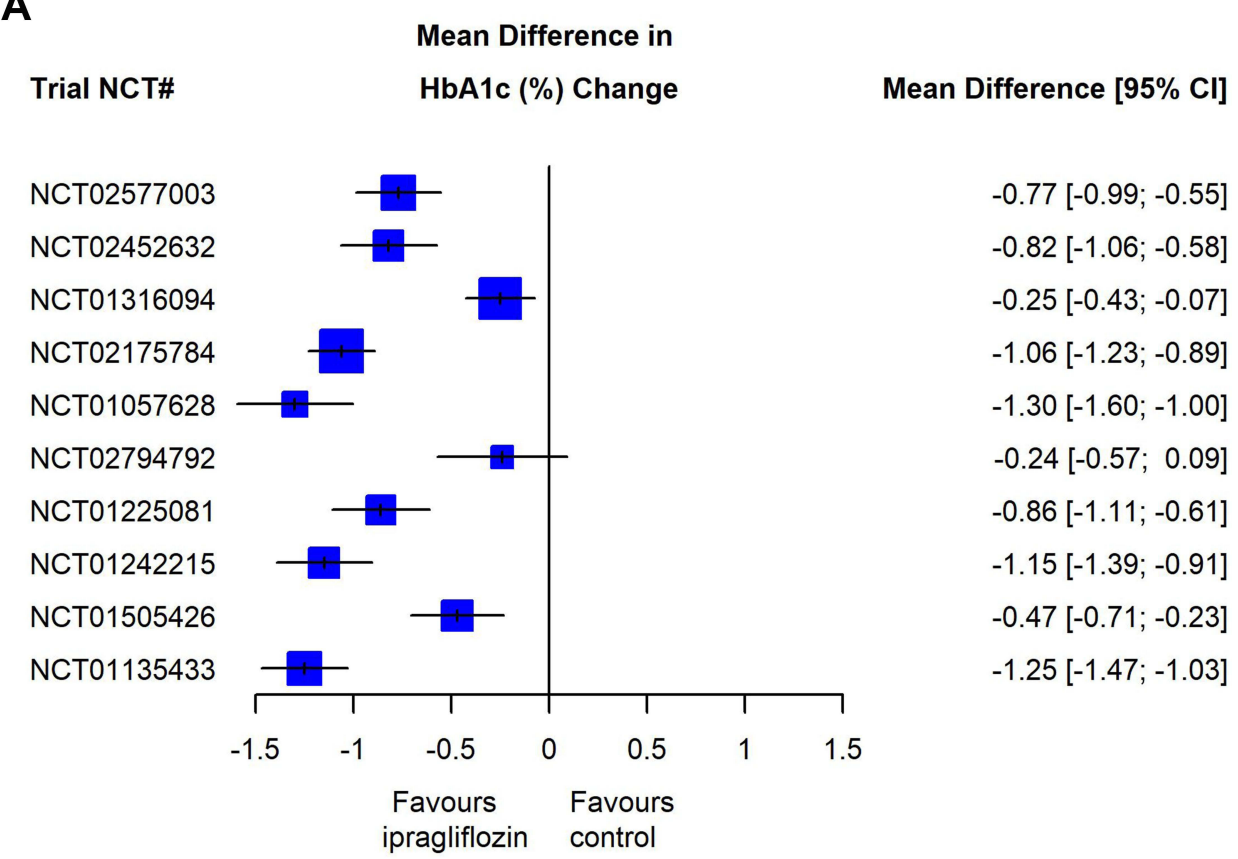

B

Mean Difference in

Trial NCT\#

FPG (mg/dL) Change

Mean Difference [95\% Cl]

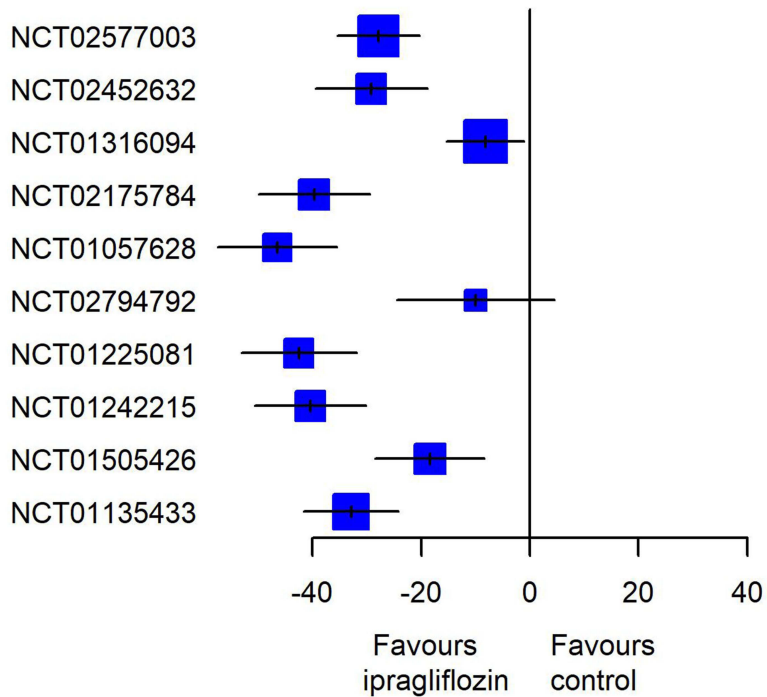

-27.90 [-35.47; -20.33]

-29.17 [-39.48; -18.86]

-8.20 [-15.32; -1.08]

-39.70 [-49.94; -29.46]

-46.50 [-57.48; -35.52]

$-10.00[-24.47 ; 4.47]$

-42.50 [-53.10; -31.90]

$-40.40[-50.64 ;-30.16]$

-18.40 [-28.43; -8.37]

-32.90 [-41.63; -24.17]

Figure 2 Forest plots for the effect of ipragliflozin on glycemic control: (A) Change in glycated hemoglobin (HbAlc) level [percent], (B) change in fasting plasma glucose (FPG) level [mg/dL].

Abbreviations: NCT\#, National Clinical Trial number; $95 \% \mathrm{Cl}, 95 \%$ confidence interval.

inhibitors have demonstrated significant reductions in cardiovascular adverse events and progression to end-stage kidney disease, in a manner that is above and beyond their glucose-lowering effect. ${ }^{50}$ This has also been corroborated by evidence from observational studies using real-world data. $^{51-53}$
Several SGLT-2 inhibitors have been associated with a reduction of the risk of hospitalization for heart failure or death from cardiovascular causes among patients with stable heart failure. ${ }^{54-56}$ Although a large trial to assess the cardioprotective role of ipragliflozin has not been conducted, evidence surrounding this can be inferred from the 


\section{A}

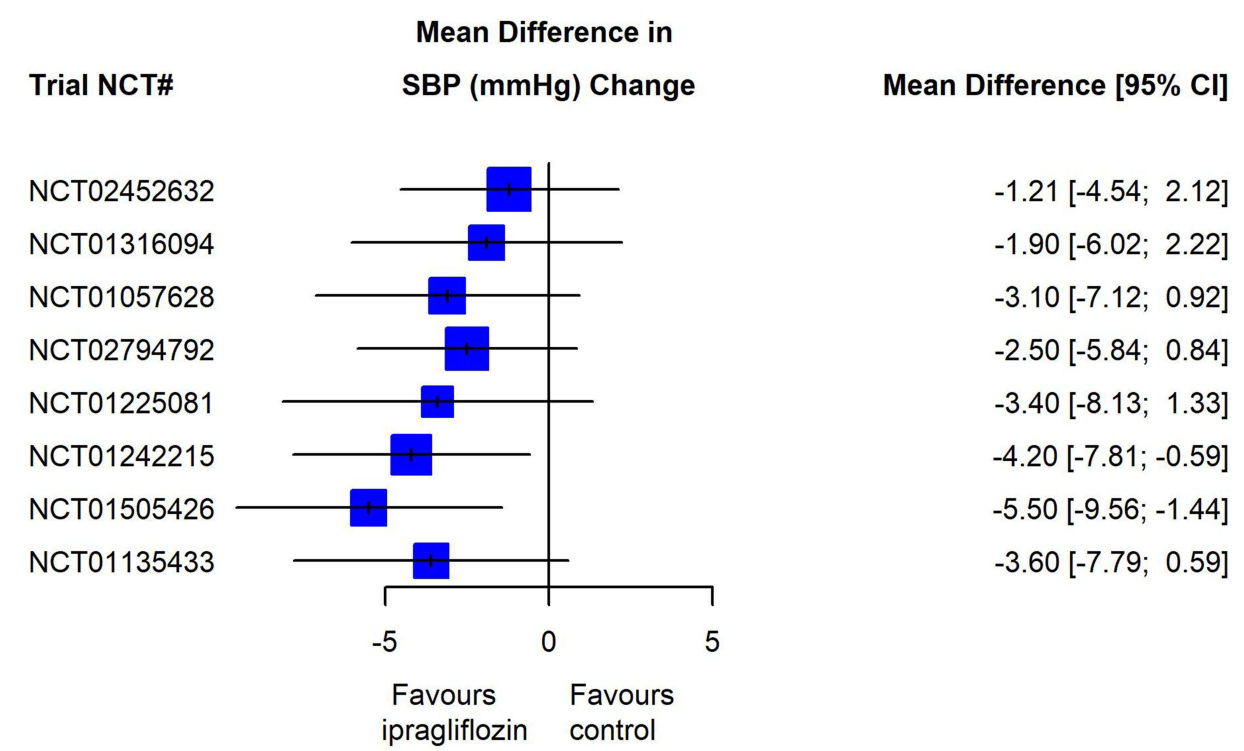

B

Mean Difference in

Trial NCT\#

DBP (mmHg) Change

Mean Difference $[95 \% \mathrm{Cl}]$

NCT02452632

NCT01316094

NCT01057628

NCT01225081

NCT01242215

NCT01505426

NCT01135433



$-1.32[-3.26 ; 0.62]$

$-1.30[-4.13 ; 1.53]$

-1.10 [-4.03; 1.83]

-5.80 [-9.12; -2.48]

-2.40 [-4.72; -0.08]

$-2.70[-5.32 ;-0.08]$

$-1.80[-4.54 ; 0.94]$

Figure 3 Forest plots for the effect of ipragliflozin on blood pressure reduction: (A) Change in systolic blood pressure (SBP) level [mmHg], (B) change in diastolic blood pressure (DBP) level $[\mathrm{mmHg}]$.

Abbreviations: NCT\#, National Clinical Trial number; $95 \% \mathrm{Cl}$, 95\% Confidence Interval.

large and international CVD-REAL 2 study, wherein ipragliflozin accounted for $8 \%$ of SGLT-2 inhibitors exposure time. $^{57}$

\section{Safety and Tolerability Considerations}

As a class, the SGLT-2 inhibitors have received much scrutiny regarding their safety profile. Multiple safety warnings have been announced by regulatory agencies across the globe. ${ }^{57-64}$ Multiple systematic reviews have assessed the safety of SGLT-2 inhibitors as a class and ipragliflozin as a molecule, either within a network meta-analysis or traditional pair-wise meta-analysis. ${ }^{7,44,65}$ We focus on the safety data for six adverse events of interest: hypoglycemia, genital tract infections, urinary tract infections, ketonerelated events, skin-related adverse events, and other notable events.

\section{Hypoglycemia}

All included trials but one ${ }^{33}$ reported on the risk of hypoglycemia associated with ipragliflozin compared to placebo. Ipragliffozin was not statistically associated with a higher risk of hypoglycemia compared to placebo by 


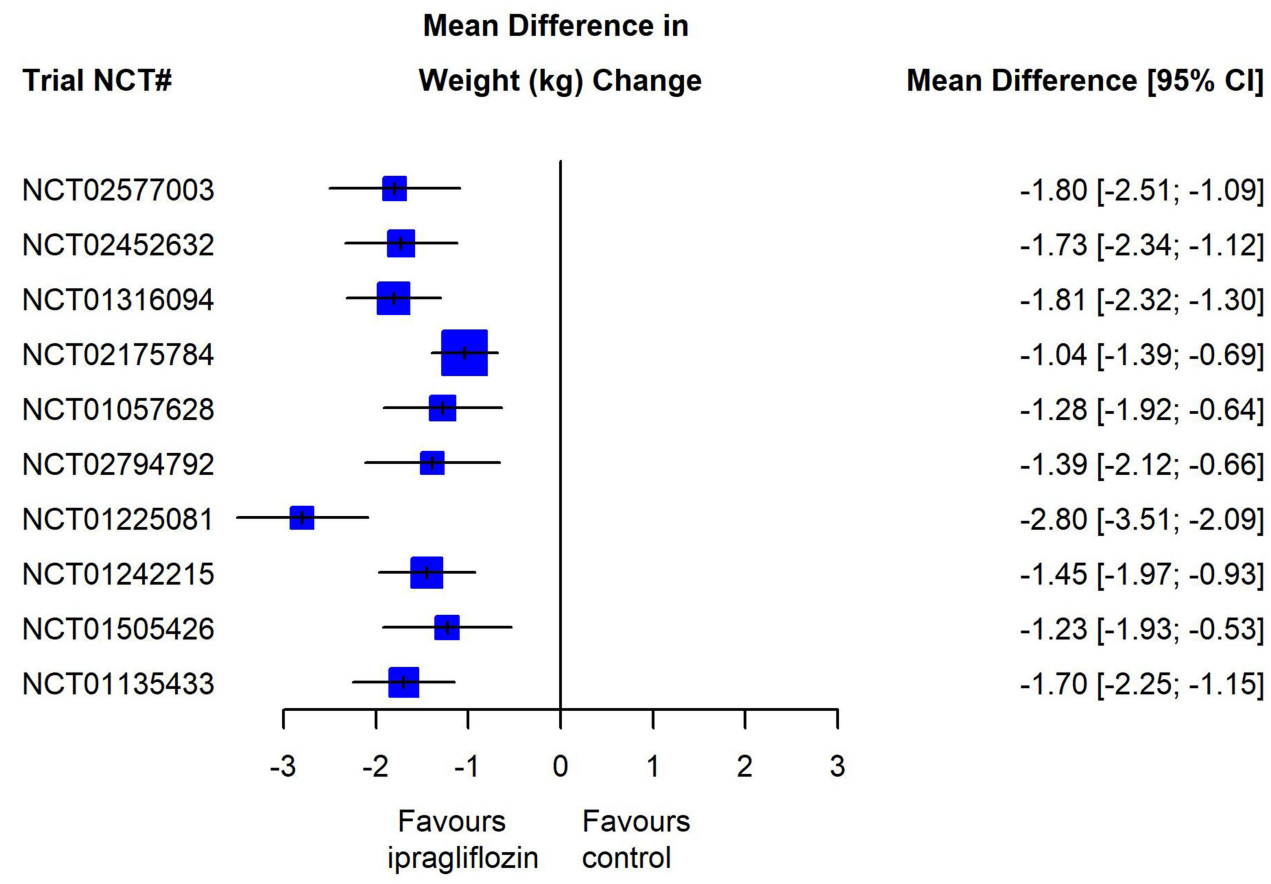

Figure 4 Forest plots for the effect of ipragliflozin on body weight reduction (kg).

Abbreviations: NCT\#, National Clinical Trial number; $95 \% \mathrm{Cl}$, 95\%confidence interval.

any of the included trials, except one in which insulin was combined with ipragliflozin ${ }^{35,44}$. This observation is consistent with previous meta-analyses. Liu et al pooled data from 8 randomized controlled trials and reported a higher risk of hypoglycemia among ipragliflozin vs placebo (pooled relative risk 1.68, 95\% CI 1.3, 2.74); however, when the trial combining ipragliflozin with insulin was excluded, there was no difference in the rate of hypoglycemia between groups (pooled relative risk 0.92, 95\% CI $0.30,2.85)$. Figure S2 Similarly, Elgebaly et al reported no differences in hypoglycemia among ipragliflozin monotherapy vs placebo but found a slight increased risk of hypoglycemia among ipragliflozin add-on therapy (pooled relative risk $1.89,95 \% \mathrm{CI} 1.15,3.10) .{ }^{41}$

\section{Genital and Urinary Tract Infections}

Compared to placebo, results from individual RCTs have not found ipragliflozin to be significantly associated with an increased risk of GTI (Figure S3). However, these trials were limited by the small number of events and thus these findings do not rule out an increased risk of GTI. ${ }^{66}$ Metaanalyses have reported an increased GTI risk associated with ipragliflozin use vs placebo. ${ }^{7,44}$ For example, Liu et al reported that genital infections were 4.5 times more likely among ipragliflozin users vs placebo. ${ }^{44}$ Additionally, evidence from larger RCTs and observational studies consistently link SGLT-2 inhibitors as a class to an increased risk of GTI. ${ }^{67-70}$ In respect to UTIs, individual RCTs do not suggest an increased risk among ipragliflozin users (Figure S4) which is corroborated by evidence from systematic reviews and meta-analyses. ${ }^{41,44}$

\section{Ketone-Related Adverse Events}

Only two of the included studies reported on the risk of ketone-related adverse events and only one trial reported at least one event (RR 2.49, 95\% CI 0.29, 20.95). This also suggests the limitation of RCTs with small sample sizes to precisely estimate the risk of rare adverse events. ${ }^{66}$ The totality of the evidence currently suggests about a doubling of risk for diabetic ketoacidosis for SGLT-2 inhibitor users compared to placebo or active comparator. ${ }^{66}$

\section{Skin Related Adverse Events}

It has been hypothesized that ipragliflozin may increase the risk of adverse skin reactions, possibly mediated through an interaction with melanin. A small hypothesis generating signal was reported using a disproportionality analysis of a spontaneous adverse event reporting database. $^{71}$ Although none of the trials estimates (Figure S5) reached statistical significance, we are not able to rule out the risk of skin-related adverse events, including rash, urticaria, pruritus and eczema associated with the use of 
ipragliflozin compared to placebo due the limits of the confidence intervals.

\section{Other Adverse Events}

Many of the most commonly reported adverse effects of ipragliflozin, as well as other SGLT-2 inhibitors, are volume depletion effects which are a downstream effect directly related to osmotic diuresis (ie, ipragliflozin's mechanism of action). Hypovolemia may lead to several sequalae including dehydration, dizziness, hypotension, pollakiuria, syncope, and thirst. The rates of volume depletion events reported in clinical trials for ipragliflozin are typically not significantly greater than placebo and are generally preventable through adequate hydration. ${ }^{44}$ The STELLA-LONG TERM was an observational study conducted in routine clinical practice over 3 years with more than 11,000 patients. Results from STELLA-LONG TERM show that the most common adverse drug reactions associated with ipragliflozin use were polyuria/pollakiuria $(5.5 \%)$ and volume depletion-events, including dehydration $(2.2 \%){ }^{72}$

Other potential adverse events of SGLT-2 inhibitors include falls and subsequent fractures due to volumedepletion related events. However, only two RCTs reported on the risk of bone fractures with only one event in each RCT (Figure S6). AKI has been another potential adverse event of interest for SGLT-2 inhibitors; however, there were no reported cases of AKI within the trials included. Other metaanalyses have not found other SGLT-2 inhibitors to be associated with an increased risk of either fracture or AKI. ${ }^{73}$ Last, none of the included trials reported any amputation events. Further research is needed to rule out an association and/or quantify a more precise association between ipragliflozin and these adverse events.

\section{Ipragliflozin in the Management of Type 2 Diabetes}

When ipragliflozin was first approved in 2014, it was indicated for T2D as monotherapy or in combination with other hypoglycemic agents, including metformin, pioglitazone, sulfonylureas, $\alpha$-glucosidase inhibitors, dipeptidyl peptidase-4 inhibitors, meglitinides, glucagon-like peptide-1 agonists or insulin. The usual adult dose is $50 \mathrm{mg}$ once daily before or after breakfast. In the context of inadequate glycemic control, the dose may be increased to $100 \mathrm{mg}$ once daily with monitoring for intolerance. The most recent Japanese Clinical Practice Guideline for Diabetes does not mention ipragliflozin specifically; however, SGLT-2 inhibitors are listed as oral hypoglycemic agents, considered after failure to reach glycemic control target on Medical nutrition therapy (MNT).$^{74}$ Additionally, the guideline highlights that the SGLT-2 inhibitors are associated with weight loss in obese patients and are less likely to be associated with hypoglycemia, however, urges clinicians to be attentive of the risk of genital tract infections, acute renal impairment and ketone body-related events. ${ }^{74}$ In 2018, ipragliflozin was also approved for T1D in combination with insulin in Japan, with a dosing regimen similar to T2D. In Korea, the use as monotherapy or in combination with metformin, pioglitazone or add-on treatment with combination of metformin and sitagliptin is approved with a recommended dose of $50 \mathrm{mg}$ once daily.

The role of SGLT-2 inhibitors in the management of T2D is well described in several prominent clinical practice guidelines. Due to their cardiorenal benefits, SGLT-2 inhibitors are recommended as an addition to metformin in case of atherosclerotic cardiovascular disease, heart failure, chronic kidney diseases, or if weight reduction is necessary. ${ }^{75,76}$

\section{Future Research Directions}

Notably, preclinical and clinical data suggest a possible role for ipragliflozin, along with other SGLT-2 inhibitors in the management on non-alcoholic fatty liver disease (NAFLD) in T2D. ${ }^{77,78}$ Thus far, only two open-label RCTs evaluated the effect of ipragliflozin in the treatment of NAFLD and found it to be significantly associated with decreased hepatosteatosis and steatohepatitis. However, larger high-quality RCTs are needed. ${ }^{77,78}$

The currently available evidence is not sufficient to draw conclusions of the specific safety profile of ipragliflozin and only that of SGLT-2 inhibitors as a class can be inferred. Additional observational studies to assess the risk of both rare and long-term adverse events of ipragliflozin are needed.

\section{Conclusions}

Ipragliflozin is a novel anti-hyperglycemic agent that lowers plasma glucose through reabsorption which is mediated through SGLT-2 blockade. Ipragliflozin is marketed in a limited number of countries on a global scale and is not approved in North America. Its use as a glucose lowering agent is modest with about a $0.5 \%$ to $1 \%$ reduction in HbAlc. However, the associated weight loss and low risk of hypoglycemia provide therapeutic advantages over other antihyperglycemic agents. Moreover, there have been no large outcome trials assessing the cardio-renal effects of ipragliflozin. Importantly, further investigation is needed to precisely assess the safety profile and long-term adverse events of ipragliflozin. 


\section{Acknowledgments}

We thank Dr. Praveen Rao Nekkar for assistance with creating Figure 1.

\section{Author Contributions}

All authors made substantial contributions to conception and design, acquisition of data, or analysis and interpretation of data; took part in drafting the article or revising it critically for important intellectual content; agreed to submit to the current journal; gave final approval of the version to be published; and agree to be accountable for all aspects of the work.

\section{Disclosure}

The authors report no conflicts of interest in this work.

\section{References}

1. American Diabetes Association. Diagnosis and classification of diabetes mellitus. Diabetes Care. 2014;37(Supp11):S81-90. doi:10.2337 dc14-S081

2. Houlden RL; Diabetes Canada Clinical Practice Guidelines Expert Committee. Diabetes Canada 2018 clinical practice guidelines for the prevention and management of diabetes in Canada. Can J Diabetes. 2018;42(Suppl 1):S1-S325. doi:10.1016/j.jcjd.2017.10.001

3. DeFronzo RA. From the triumvirate to the "ominous octet": a new paradigm for the treatment of type 2 diabetes mellitus. Diabetes. 2009;58(4):773-795. doi:10.2337/db09-9028

4. Kalra S. Sodium Glucose Co-Transporter-2 (SGLT2) inhibitors: a review of their basic and clinical pharmacology. Diabetes Ther. 2014;5(2):355-366. doi:10.1007/s13300-014-0089-4

5. Haas B, Eckstein N, Pfiefer V, et al. Efficacy, safety and regulatory status of SGLT2 inhibitors: focus on canagliflozin. Nutr Diabetes. 2014;4:e143. doi:10.1038/nutd.2014.40

6. Astellas Pharma Inc. Approval of suglat ${ }^{\circledR}$ tablets, a selective SGLT2 inhibitor for treatment of type 2 diabetes, in Japan [press release]; 2014. Available from: https://www.astellas.com/en/news/11351. Accessed March 12, 2021.

7. Kashiwagi A, Shestakova MV, Ito Y, et al. Safety of ipragliflozin in patients with type 2 diabetes mellitus: pooled analysis of phase II/III/ IV clinical trials. Diabetes Ther. 2019;10(6):2201-2217. doi:10.1007/ s13300-019-00699-8

8. Covidence systematic review software, Veritas Health Innovation, Melbourne, Australia; 2021. Available from: www.covidence.org. Accessed June 26, 2021.

9. Committee on the Proper Use of SGLT2 Inhibitors. Recommendations on the proper use of SGLT2 inhibitors. J Diabetes Investig. 2020;11(1):257-261. doi:10.1111/jdi.13160

10. Imamura M, Nakanishi K, Suzuki T, et al. Discovery of ipragliflozin (ASP1941): a novel C-glucoside with benzothiophene structure as a potent and selective sodium glucose co-transporter 2 (SGLT2) inhibitor for the treatment of type 2 diabetes mellitus. Bioorgan Med Chem. 2012;20(10):3263-3279. doi:10.1016/j.bmc.2012.03.051

11. Tahara A, Kurosaki E, Yokono M, et al. Effects of SGLT2 selective inhibitor ipragliflozin on hyperglycemia, hyperlipidemia, hepatic steatosis, oxidative stress, inflammation, and obesity in type 2 diabetic mice. Eur J Pharmacol. 2013;715(1-3):246-255. doi:10.1016/j. ejphar.2013.05.014

12. Tiwari S, Wadher S, Fartade S, Vikhar C. Gliflozin a new class for type-ii diabetes mellitus: an overview. IJPSR. 2019;10(9):4070-4077.
13. Cayman Chemical. Product information: ipragliflozin; 2020. Available from: https://www.caymanchem.com/pdfs/22287. Accessed March 12, 2021.

14. Kadokura T, Akiyama N, Kashiwagi A, et al. Pharmacokinetic and pharmacodynamic study of ipragliflozin in Japanese patients with type 2 diabetes mellitus: a randomized, double-blind, placebo-controlled study. Diabetes Res Clin Pract. 2014;106:50-56. doi:10.1016/j.diabres.2014.07.020

15. Schwartz SL, Akinlade B, Klasen S, et al. Safety, pharmacokinetic, and pharmacodynamic profiles of ipragliflozin (ASP1941), a novel and selective inhibitor of sodium-dependent glucose co-transporter 2, in patients with type 2 diabetes mellitus. Diabetes Technol Ther. 2011;13:1219-1227. doi:10.1089/dia.2011.0012

16. Kadokura T, Zhang W, Krauwinkel W, et al. Clinical pharmacokinetics and pharmacodynamics of the novel SGLT2 inhibitor ipragliflozin. Clin Pharmacokinet. 2014;53(11):975-988. doi:10.10 07/s40262-014-0180-z

17. Saito M, Kaibara A, Kadokura T, et al. Pharmacokinetic and pharmacodynamic modelling for renal function dependent urinary glucose excretion effect of ipragliflozin, a selective sodium-glucose cotransporter 2 inhibitor, both in healthy subjects and patients with type 2 diabetes mellitus. Br J Clin Pharmacol. 2019;85(8):1808-1819. doi:10.1111/bcp. 13972

18. Smulders R, Ishikawa H, Nakajo I, et al. The effect of renal impairment on the pharmacokinetics and urinary glucose excretion of the SGLT2 inhibitor ipragliflozin (ASP1941) in Japanese type 2 diabetes mellitus patients. Poster presentation at the 47th Annual Meeting of the European Association for the Study of Diabetes (EASD); September 12-16, 2011; Lisbon, Portugal; Abstract Number: 847.

19. Veltkamp SA, van Dijk J, Krauwinkel W, et al. The effect of renal impairment on the pharmacokinetics and urinary glucose excretion of the SGLT2 inhibitor ipragliflozin (ASP1941) in type2 diabetes mellitus patients. Poster presentation at the 71st Sci-entific Sessions of the American Diabetes Association (ADA); June 24-28, 2011, San Diego, CA; Abstract Number: 1127-P.

20. Ferrannini E, Veltkamp SA, Smulders RA, et al. Renal glucose handling: impact of chronic kidney disease and sodium-glucose cotransporter 2 inhibition in patients with type 2 diabetes. Diabetes Care. 2013;36:1260-1265. doi:10.2337/dc12-1503

21. Bailey CJ. Renal glucose reabsorption inhibitors to treat diabetes. Trends Pharmacol Sci. 2011;32(2):63-71. doi:10.1016/j.tips.2010. 11.011

22. Wright EM. Renal Na+-glucose cotransporters. Am J Physiol Renal Physiol. 2001;280(1):F10-8. doi:10.1152/ajprenal.2001.280.1.F10

23. Bakris GL, Fonseca VA, Sharma K, Wright EM. Renal sodiumglucose transport: role in diabetes mellitus and potential clinical implications. Kidney Int. 2009;75(12):1272-1277. doi:10.1038/ ki.2009.87

24. Mogensen CE. Glomerular filtration rate and renal plasma flow in normal and diabetic man during elevation of blood sugar levels. Scand J Clin Lab Invest. 1971;28(2):177-182. doi:10.3109/00365 517109086898

25. Vallon V, Sharma K. Sodium-glucose transport: role in diabetes mellitus and potential clinical implications. Curr Opin Nephrol Hypertens. 2010;19(5):425-431. doi:10.1097/MNH.0b013e32833 bec06

26. Mather A, Pollock C. Glucose handling by the kidney. Kidney Int. 2011;79:S1-6. doi:10.1038/ki.2010.509

27. Ferrannini E. Sodium-glucose co-transporters and their inhibition: clinical physiology. Cell Metab. 2017;26(1):27-38. doi:10.1016/j. cmet.2017.04.011

28. Gallo LA, Wright EM, Vallon V. Probing SGLT2 as a therapeutic target for diabetes: basic physiology and consequences. Diab Vasc Dis Res. 2015;12(2):78-89. doi:10.1177/1479164114561992 
29. Liu JJ, Lee T, DeFronzo RA. Why do SGLT2 inhibitors inhibit only $30-50 \%$ of renal glucose reabsorption in humans? Diabetes. 2012;61 (9):2199-2204. doi:10.2337/db12-0052

30. Zhang W, Krauwinkel WJ, Keirns J, et al. The effect of moderate hepatic impairment on the pharmacokinetics of ipragliflozin, a novel sodium glucose co-transporter 2 (SGLT2) inhibitor. Clin Drug Investig. 2013;33:489-496.

31. Han KA, Chon S, Chung CH, et al. Efficacy and safety of ipragliflozin as an add-on therapy to sitagliptin and metformin in Korean patients with inadequately controlled type 2 diabetes mellitus: a randomized controlled trial. Diabetes Obes Metab. 2018;20 (10):2408-2415. doi:10.1111/dom.13394

32. Kashiwagi A, Kazuta K, Takinami Y, Yoshida S, Utsuno A, Nagase I. Ipragliflozin improves glycemic control in Japanese patients with type 2 diabetes mellitus: the BRIGHTEN study. Diabetol Int. 2015;6(1):8-18. doi:10.1007/s13340-014-0164-0

33. ClinicalTrials.gov. Identifier NCT02577003, double-blind ipragliflozin add-on study in Japanese participants with type 2 diabetes mellitus who have inadequate glycemic control on sitagliptin (MK-0431J-843); 2021. Bethesda (MD): National Library of Medicine (US). Available from: https://clinicaltrials.gov/ct2/show/NCT02577003. Accessed March 12, 2021.

34. Kashiwagi A, Takahashi H, Ishikawa $\mathrm{H}$, et al. A randomized, double-blind, placebo-controlled study on long-term efficacy and safety of ipragliflozin treatment in patients with type 2 diabetes mellitus and renal impairment: results of the long-term ASP1941 safety evaluation in patients with type 2 diabetes with renal impairment (LANTERN) study. Diabetes Obes Metab. 2015;17 (2):152-160. doi:10.1111/dom.12403

35. Astellas Pharma Inc. Postmarketing clinical study of ipragliflozindouble-blind, parallel-group study in combination with insulin in patients with type 2 diabetes mellitus; 2016. Available from: https:// astellasclinicalstudyresults.com/hcp/study.aspx?ID=171. Accessed March 12, 2021.

36. Shestakova MV, Wilding JPH, Wilpshaar W, Tretter R, Orlova VL, Verbovoy AF. A phase 3 randomized placebo-controlled trial to assess the efficacy and safety of ipragliflozin as an add-on therapy to metformin in Russian patients with inadequately controlled type 2 diabetes mellitus. Diabetes Res Clin Pract. 2018;146:240-250. doi:10.1016/j.diabres.2018.10.018

37. Kashiwagi A, Shiga T, Akiyama N, et al. Efficacy and safety of ipragliflozin as an add-on to pioglitazone in Japanese patients with inadequately controlled type 2 diabetes: a randomized, double-blind, placebo-controlled study (the SPOTLIGHT study). Diabetol Int. 2015;6(2):104-116. doi:10.1007/s13340-014-0182-y

38. Kashiwagi A, Akiyama N, Shiga T, et al. Efficacy and safety of ipragliflozin as an add-on to a sulfonylurea in Japanese patients with inadequately controlled type 2 diabetes: results of the randomized, placebo-controlled, double-blind, phase III EMIT study. Diabetol Int. 2015;6:125-138. doi:10.1007/s13340-014-0184-9

39. Lu CH, Min KW, Chuang LM, Kokubo S, Yoshida S, Cha BS. Efficacy, safety, and tolerability of ipragliflozin in Asian patients with type 2 diabetes mellitus and inadequate glycemic control with metformin: results of a phase 3 randomized, placebo-controlled, double-blind, multicenter trial. $J$ Diabetes Investig. 2016;7 (3):366-373. doi:10.1111/jdi.12422

40. Kashiwagi A, Kazuta K, Goto K, Yoshida S, Ueyama E, Utsuno A. Ipragliflozin in combination with metformin for the treatment of Japanese patients with type 2 diabetes: ILLUMINATE, a randomized, double-blind, placebo-controlled study. Diabetes Obes Metab. 2015;17(3):304-308. doi:10.1111/dom.12331

41. Elgebaly A, Abdelazeim N, Abdelazeim B, et al. Tolerability and efficacy of ipragliflozin in the management of inadequately controlled type 2 diabetes mellitus: a systematic review and meta-analysis. Exp Clin Endocrinol Diabetes. 2021;129(1):56-72. doi:10.1055/a-0579-7860
42. Kashiwagi A, Yoshida S, Kawamuki K, et al. Effects of ipragliflozin, a selective sodium-glucose co-transporter 2 inhibitor, on blood pressure in Japanese patients with type 2 diabetes mellitus: a pooled analysis of six randomized, placebo-controlled clinical trials. Diabetol Int. 2016;8(1):76-86. doi:10.1007/s13340-016-0283-x

43. Kashiwagi A, Sakatani T, Nakamura I, et al. Improved cardiometabolic risk factors in Japanese patients with type 2 diabetes treated with ipragliflozin: a pooled analysis of six randomized, placebo-controlled trials. Endocr J. 2018;65(7):693-705. doi:10. 1507/endocrj.EJ17-0491

44. Liu D, Chen H, Song F, Ahmed MA, Wu H. Adverse drug events observed with the novel sodium/glucose co-transporter 2 inhibitor ipragliflozin for the treatment of patients with type 2 diabetes mellitus: a systematic review and meta-analysis of randomized studies. Adv Ther. 2020;37(10):4356-4369. doi:10.1007/s12325-020-01471-2

45. Zinman B, Wanner C, Lachin JM, et al.; EMPA-REG OUTCOME Investigators. Empagliflozin, cardiovascular outcomes, and mortality in type 2 diabetes. $N$ Engl J Med. 2015;373(22):2117-2128. doi:10.1056/NEJMoa1504720

46. Neal B, Perkovic V, Mahaffey KW, et al.; CANVAS Program Collaborative Group. Canagliflozin and cardiovascular and renal events in type 2 diabetes. $N$ Engl J Med. 2017;377(7):644-657. doi:10.1056/NEJMoa1611925

47. Wiviott SD, Raz I, Bonaca MP; DECLARE-TIMI 58 Investigators. Dapagliflozin and cardiovascular outcomes in type 2 diabetes. $N$ Engl J Med. 2019;380(4):347-357. doi:10.1056/NEJMoa1812389

48. Cannon CP, Pratley R, Dagogo-Jack S, et al.; VERTIS CV Investigators. Cardiovascular outcomes with ertugliflozin in type 2 diabetes. $N$ Engl J Med. 2020;383(15):1425-1435. doi:10.1056/ NEJMoa2004967

49. Bhatt DL, Szarek M, Pitt B, et al.; SCORED Investigators. Sotagliflozin in patients with diabetes and chronic kidney disease. N Engl J Med. 2021;384(2):129-139. doi:10.1056/NEJMoa2030186

50. Rangaswami J, Bhalla V, de Boer IH, et al. Cardiorenal protection with the newer antidiabetic agents in patients with diabetes and chronic kidney disease: a scientific statement from the American Heart Association. Circulation. 2020;142(17):e265-e286. doi:10. 1161/CIR.0000000000000920

51. Pasternak B, Wintzell V, Melbye M, et al. Use of sodium-glucose co-transporter 2 inhibitors and risk of serious renal events: Scandinavian cohort study. BMJ. 2020;369:m1186. doi:10.1136/bmj. m1186

52. Heerspink HJL, Karasik A, Thuresson M, et al. Kidney outcomes associated with use of SGLT2 inhibitors in real-world clinical practice (CVD-REAL 3): a multinational observational cohort study. Lancet Diabetes Endocrinol. 2020;8(1):27-35. doi:10.1016/S22138587(19)30384-5

53. Filion KB, Lix LM, Yu OH, et al.; Canadian Network for Observational Drug Effect Studies (CNODES) Investigators. Sodium glucose cotransporter 2 inhibitors and risk of major adverse cardiovascular events: multi-database retrospective cohort study. BMJ. 2020;370:m3342. doi:10.1136/bmj.m3342

54. Packer M, Anker SD, Butler J, et al. Cardiovascular and renal outcomes with empagliflozin in heart failure. $N$ Engl J Med. 2020;383 (15):1413-1424. doi:10.1056/NEJMoa2022190

55. McMurray JJV, Solomon SD, Inzucchi SE, et al. Dapagliflozin in patients with heart failure and reduced ejection fraction. $N$ Engl J Med. 2019;381(21):1995-2008. doi:10.1056/NEJMoa1911303

56. Bhatt DL, Szarek M, Steg PG, et al. Sotagliflozin in patients with diabetes and recent worsening heart failure. $N$ Engl J Med. 2021;384 (2):117-128. doi:10.1056/NEJMoa2030183

57. CVD-REAL Investigators and Study Group; Kosiborod M, Lam CSP, Kohsaka S, et al. Cardiovascular events associated with SGLT-2 inhibitors versus other glucose-lowering drugs: the CVD-REAL 2 study. J Am Coll Cardiol. 2018;71(23):2628-2639. doi:10.1016/j. jacc.2018.03.009. 
58. MedEffect Canada. Summary safety review-sodium glucose cotransporter 2 (SGLT-2) inhibitors INVOKANA (canagliflozin) and FORXIGA (dapagliflozin)-evaluation of a potential risk of acute kidney injury; 2019. Available from: https://www.canada.ca/en/ health-canada/services/drugs-health-products/medeffect-canada /safety-reviews/summary-safety-review-sodium-glucosecotransporter-2-sglt2-inhibitors-invokana-canagliflozin-forxigadapagliflozinl-risk.html. Accessed March 12, 2021.

59. MedEffect Canada. Summary safety review-SGLT2 inhibitors (canagliflozin, dapagliflozin, empagliflozin)-assessing the potential risk of bone-related side effects; 2016. Available from: https:/www.canada. ca/en/health-canada/services/drugs-health-products/medeffect-canada /safety-reviews/summary-safety-review-sglt2-inhibitors-canagliflozin -dapagliflozin-empagliflozin-risk-bone.html. Accessed March 12, 2021.

60. MedEffect Canada. Summary safety review-SGLT-2 inhibitors (canagliflozin, dapagliflozin, empagliflozin)-assessing the risk of the body producing high levels of acids in the blood (diabetic ketoacidosis); 2016. Available from: https://www.canada.ca/en/healthcanada/ser vices/drugs-health-products/medeffect-canada/safety-reviews/sum mary-safetyreview-sglt2-inhibitors-canagliflozin-dapagliflozinempagliflozin.html. Accessed March 12, 2021.

61. US Food and Drug Administration. FDA confirms increased risk of leg and foot amputations with the diabetes medicine canagliflozin (Invokana, Invokamet, Invokamet XR); 2017. Available from: https:// www.fda.gov/media/104870/download. Accessed March 12, 2021.

62. US Food and Drug Administration. FDA revises labels of SGLT2 inhibitors for diabetes to include warnings about too much acid in the blood and serious urinary tract infections; 2015. Available from: https:// www.fda.gov/media/94822/download. Accessed March 12, 2021.

63. European Medicines Agency. SGLT2 inhibitors: PRAC makes recommendations to minimise risk of diabetic ketoacidosis; 2016. Available from: https:/www.ema.europa.eu/en/documents/referral/sglt2inhibitors-article-20-procedure-prac-makes-recommendationsminimise-risk-diabetic-ketoacidosis_en.pdf. Accessed March 12, 2021.

64. Pharmaceuticals and Medical Devices Agency. Pharmaceuticals and medical devices safety information; 2015. Available from: https:// www.pmda.go.jp/files/000208624.pdf. Accessed March 12, 2021.

65. Donnan JR, Grandy CA, Chibrikov E, et al. Comparative safety of the sodium glucose co-transporter 2 (SGLT2) inhibitors: a systematic review and meta-analysis. BMJ Open. 2019;9(1):e022577. doi:10.1136/bmjopen-2018-022577

66. Alkabbani W, Pelletier RT, Gamble JM. Sodium glucose co-transporter-2 (SGLT2) inhibitors and the risk of diabetic ketoacidosis: an example of complementary evidence for rare adverse events. Am J Epidemiol. 2021. doi:10.1093/aje/kwab052

67. Li D, Wang T, Shen S, Fang Z, Dong Y, Tang H. Urinary tract and genital infections in patients with type 2 diabetes treated with sodium-glucose co-transporter 2 inhibitors: a meta-analysis of randomized controlled trials. Diabetes Obes Metab. 2017;19(3):348-355. doi:10.1111/dom. 12825
68. Liu J, Li L, Li S, et al. Effects of SGLT2 inhibitors on UTIs and genital infections in type 2 diabetes mellitus: a systematic review and meta-analysis. Sci Rep. 2017;7(1):2824. doi:10.1038/s41598-01702733-w

69. Dave CV, Schneeweiss S, Patorno E. Comparative risk of genital infections associated with sodium-glucose co-transporter-2 inhibitors. Diabetes Obes Metab. 2019;21(2):434-438. doi:10.1111/dom.13531

70. Lega IC, Bronskill SE, Campitelli MA, et al. Sodium glucose cotransporter 2 inhibitors and risk of genital mycotic and urinary tract infection: a population-based study of older women and men with diabetes. Diabetes Obes Metab. 2019;21(11):2394-2404. doi:10.11 11/dom. 13820

71. Sakaeda T, Kobuchi S, Yoshioka R, et al. Susceptibility to serious skin and subcutaneous tissue disorders and skin tissue distribution of sodium-dependent glucose co-transporter type 2 (SGLT2) inhibitors. Int J Med Sci. 2018;15(9):937-943. doi:10.7150/ijms.22224

72. Nakamura I, Maegawa H, Tobe K, Uno S. Real-world evidence for long-term safety and effectiveness of ipragliflozin in Japanese patients with type 2 diabetes mellitus: final results of a 3-year post-marketing surveillance study (STELLA-LONG TERM). Expert Opin Pharmacother. 2021;22(3):373-387. doi:10.1080/14656566.20 20.1817388

73. Pelletier R, Ng K, Alkabbani W, Labib Y, Mourad N, Gamble JM. Adverse events associated with sodium glucose co-transporter 2 inhibitors: an overview of quantitative systematic reviews. Ther $A d v$ Drug Saf. 2021;12:2042098621989134. doi:10.1177/2042098621 989134

74. Araki E, Goto A, Kondo T, et al. Japanese clinical practice guideline for diabetes 2019. J Diabetes Investig. 2020;11(4):1020-1076. doi:10.1111/jdi.13306

75. American Diabetes Association. 9. Pharmacologic approaches to glycemic treatment: standards of medical care in diabetes-2021. Diabetes Care. 2021;44(Supplement 1):S111-24. doi:10.2337/dc21S009

76. Lipscombe L, Butalia S, Dasgupta K, et al. Pharmacologic glycemic management of type 2 diabetes in adults: 2020 update. Can $J$ Diabetes. 2020;44(7):575-591. doi:10.1016/j.jcjd.2020.08.001

77. Ito $\mathrm{D}$, Shimizu $\mathrm{S}$, Inoue $\mathrm{K}$, et al. Comparison of ipragliflozin and pioglitazone effects on nonalcoholic fatty liver disease in patients with type 2 diabetes: a randomized, 24-week, open-label, active-controlled trial. Diabetes Care. 2017;40(10):1364-1372. doi: $10.2337 / \mathrm{dc} 17-0518$

78. Han E, Lee Y-H, Lee B-W, Kang ES, Cha B-S. Ipragliflozin additively ameliorates non-alcoholic fatty liver disease in patients with type 2 diabetes controlled with metformin and pioglitazone: a 24-week randomized controlled trial. J Clin Med. 2020;9(1):259. doi:10.3390/jcm9010259

\section{Publish your work in this journal}

Drug Design, Development and Therapy is an international, peerreviewed open-access journal that spans the spectrum of drug design and development through to clinical applications. Clinical outcomes, patient safety, and programs for the development and effective, safe, and sustained use of medicines are a feature of the journal, which has also been accepted for indexing on PubMed Central. The manuscript management system is completely online and includes a very quick and fair peer-review system, which is all easy to use. Visit http://www. dovepress.com/testimonials.php to read real quotes from published authors. 